\title{
Operation and Calibration of a Highly Granular Hadron Calorimeter with SiPM-on-Tile Read-out
}

\author{
O. Pinto for the CALICE Collaboration
}

\begin{abstract}
The Analogue Hadron Calorimeter (AHCAL) is being developed within the CALICE collaboration for experiments at a future lepton collider. It is a sampling calorimeter with alternating layers of steel absorber plates and plastic scintillator tiles as active material. In the SiPM on tile design, the tiles are directly coupled to silicon photomultipliers (SiPMs). The front-end electronics are integrated into the active layers of the calorimeter. They are designed for low power consumption by rapidly power cycling according to the beam structure of a linear accelerator. In 2017 and 2018, a new large prototype with 38 active layers of $72 \times 72 \mathrm{~cm}^{2}$ size has been built. Each active layer consists of four readout boards with four 36 channel SPIROC2E readout ASIC each, resulting in 576 channels per layer. The prototype has been designed for mass production and assembly techniques: It uses injection-moulded tiles which were wrapped semi-automatically in reflector foil, tiles and electronics components were assembled using pick-and-place machines and all detector parts were tested during assembly. The prototype with $\sim 22,000$ channels was commissioned at DESY and took muon, electron and pion data at the CERN SPS to demonstrate the capabilities of a SiPM-on-tile calorimeter concept with scalable detector design and the reliable operation of a large prototype. This proceedings gives an overview of the calibration results of the large CALICE AHCAL technological prototype.
\end{abstract}

\section{INTRODUCTION}

$\mathbf{T}$ HE physics at future high-energy lepton colliders demands a jet energy reconstruction with unprecedented precision. It can be reached by employing Particle Flow Algorithms (PFA)[1], which are capable of achieving this by measuring each particle in the detector component with the best energy resolution. These algorithms require highly granular calorimeters to deliver optimal performance in jet energy resolution of the order of $3-4 \%$ for jet energies between 45 $\mathrm{GeV}$ and several $100 \mathrm{GeV}$. Within the CALICE collaboration several highly granular technologies for electromagnetic and hadronic calorimeters are developed. One of these technologies is the Analog Hadron Calorimeter (AHCAL)[2].

\section{The CALICE SiPM-ON-TILE HADRon CALORIMETER TECHNOLOGICAL PROTOTYPE}

The AHCAL technological prototype consists of a nonmagnetic stainless steel absorber structure with approximately $17.2 \mathrm{~mm}$ thick plates and 38 active layers which are placed

Manuscript received December 13, 2019. This project has received funding from the European Union Horizon 2020 Research and Innovation programme under Grant Agreement no. 654168.

O. Pinto is with the Deutsches Elektronen-Synchrotron (DESY), Germany (e-mail: olin.pinto@desy.de)

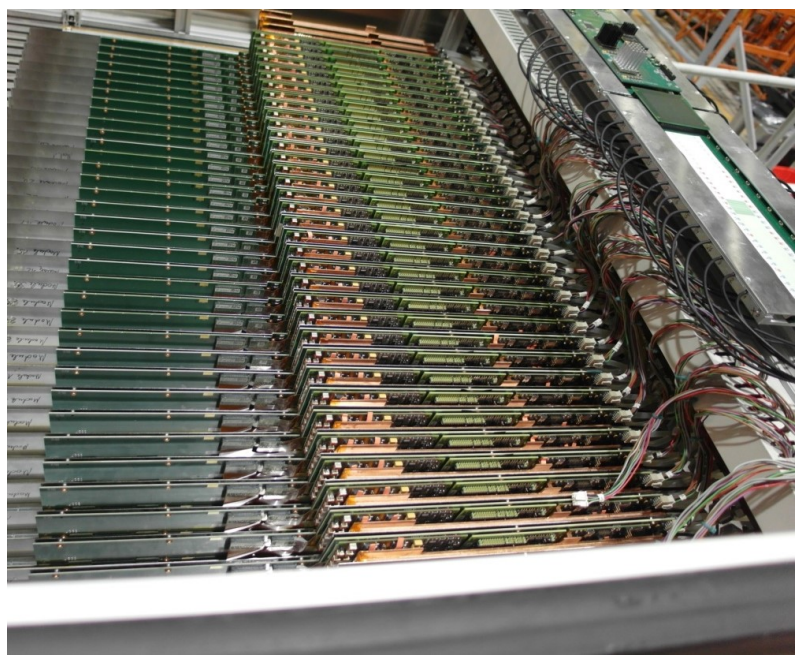

Fig. 1. Top view of AHCAL technological prototype fully assembled with 38 active layers

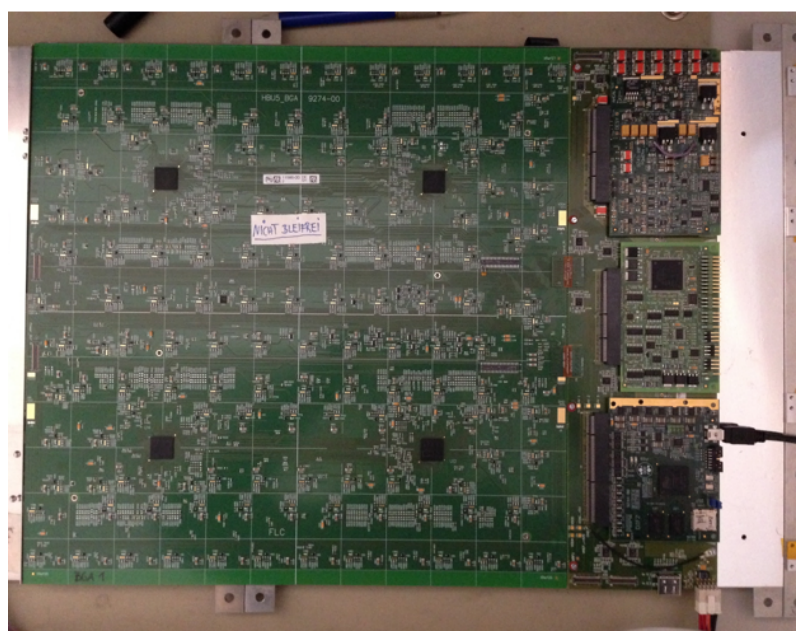

Fig. 2. HCAL Base Unit.

in the absorber structure as shown in Fig 1. It has 21,888 channels read out on an individual basis by SiPMs. The basic unit of the active elements is the HCAL Base Unit (HBU)[3], which has a size of $36 \times 36 \mathrm{~cm}^{2}$. It is equipped with $144 \mathrm{SiPMs}$ which are read out by four SPIROC2E ASICs[4]. The key features of the electronics are its integration into the active volume and its capability to operate in power-pulsed mode which reduces the power consumption and eliminate the need 
for active cooling by exploiting the beam structure of linear colliders. The SPIROC2E ASIC also provides a cell-by-cell auto trigger and individual hit time with a precision of a few ns in test beam operations. In linear collider operation conditions with shorter data-taking windows, sub-ns time resolution is expected. The prototype is constructed with injection-moulded polystyrene scintillator tiles[5] with a dimple at the centre optimised for best light collection[6]. They are read out by Hamamatsu MPPC S13360-1325PE photon sensors. The technological prototype has a layout scalable to full a ILC detector.

\section{CALIBRATION}

The calibration procedure of the AHCAL prototype has several goals:

- The conversion of the hit energy and time from electronics to physical units

- if necessary, the equalisation of the cell response to allow an efficient auto-triggering in all channels

- the monitoring of the stability of the detector

A global calibration chain is necessary to properly calibrate the calorimeter channels. Every channel has to be calibrated separately due to non-uniformities such as unequal tile wrapping, glueing or SiPM and ASIC specific effects. Two calibration chains are needed, one for energy calibration and the other for time calibration. The hit energy or SiPM charge is readout as ADC (Analogue to Digital Converter) and needs to be calibrated in units of minimum ionising particles (MIPs). The time is stored as a charge TDC (Time to Digital Converter) and is calibrated into values of nanoseconds.

The calibrated hit energy $E_{i}$ in units of MIP can be determined by eq. 1 .

$$
E_{i}[M I P]=f_{\text {desat }}(\text { pixels }) \frac{\left(A_{i}[A D C]-\text { Pedestal }\right) / I C}{M I P}
$$

where

$$
\text { pixels }=\frac{\left(A_{i}[A D C]-\text { Pedestal }\right) / I C}{\text { Gain }}
$$

the function $\mathrm{f}_{\text {desat }}$ is the inverse of the SiPM response function, describing the SiPM output signal as a function of the incoming light intensity. At small amplitudes $\mathrm{f}_{\text {desat }}$ is close to one and increases strongly for large signals. IC is the inter-gain calibration.

As a final step in calibration, cluster energies will be converted to the $\mathrm{GeV}$ scale using electron testbeam data of known energies.

\section{A. Gain Calibration}

The gain calibration allows the possibility of monitoring the detector performance and stability. The gain calibration is performed using dedicated LED runs. For all the AHCAL channels, single photon spectra are taken at low intensity LED light, and are fitted with a multi-Gaussian function. The gain value is extracted as the distance between the first and the second single photon-electron peaks. An example of a typical single photon spectrum is shown in Fig 3 . The distance between two neighbouring peaks is left as a single free parameter in the multi-Gaussian fit. The width of the peaks is dominated by electronic noise. The statistical uncertainty on the gain determination is less than $1 \%$ for fits which pass quality criteria.

During testbeam operation LED runs were taken approximately once per day. Gain extraction was achieved for $95 \%$ of the channels. The gain values for the remaining $5 \%$ of channels were taken as the average of the channels of the corresponding chip. Combining several gain runs, more than $99.9 \%$ of all channels can be calibrated individually. The mean gain is 16.6 ADC/pixel with a spread of $1.0 \mathrm{ADC} /$ pixel which is about $6 \%$ as shown in Fig 4 . For channels on the same ASIC the spread is only $2.5 \%$, showing that the gain variation between ASICs contributes significantly to the overall spread. The gain is consistent between the May 2018 run and the June 2018 run (both without power pulsing). For the October 2018 run (with power pulsing) a small shift in gain is observed which was caused by electronic effects.

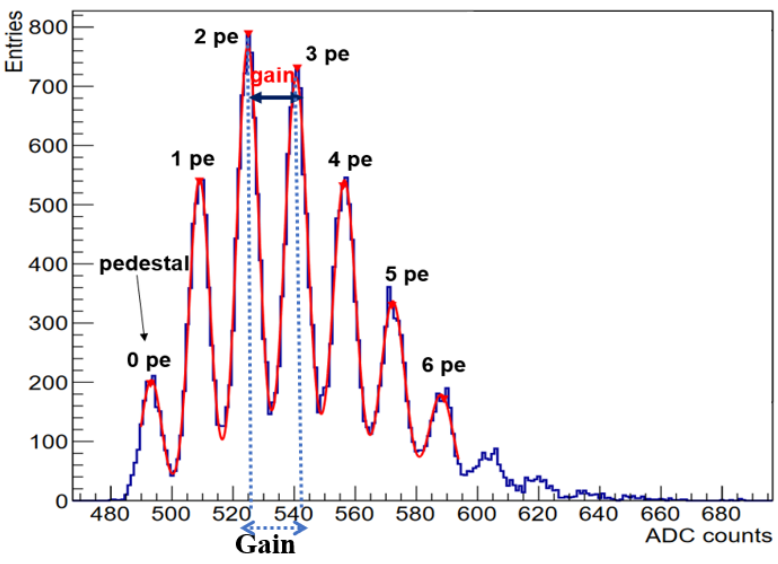

Fig. 3. SiPM single photoelectron spectrum of a single channel.

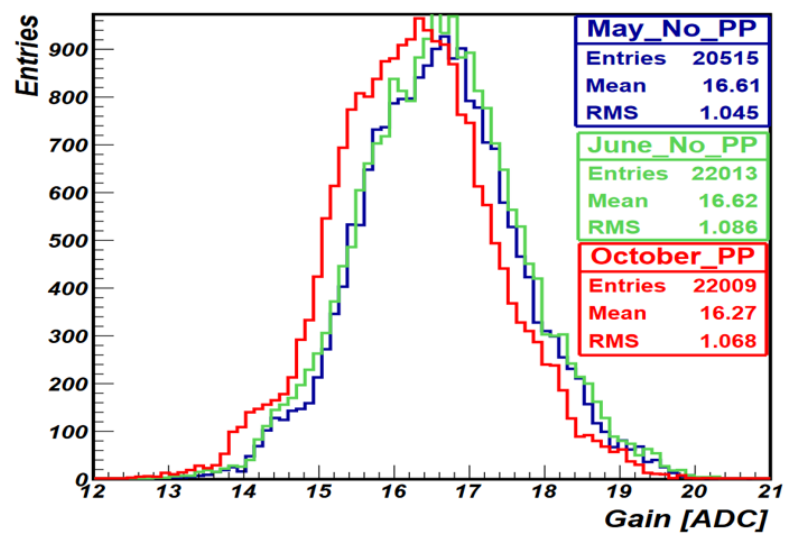

Fig. 4. Gain constant extracted for all AHCAL channels.

\section{B. MIP Calibration}

Minimum-ionising particles (MIPs) are used to perform a cell to cell equalization. For each channel the measured hit energy spectrum for muons is analysed. Its most probable value is derived from a fit with a Landau function convoluted with a 
Gaussian. An example of a single channel is shown in Fig 5 The fit function takes into account not only the energy loss of muons within the scintillator tiles, but also contributions from photon counting statistics and electronic noise. The MIP calibration efficiency, i.e. the amount of successful fits of muon hit energy spectra, is about $99.92 \%$. As shown in Fig 6 the mean MIP calibration constant is 217 ADC with an RMS spread of 30 ADC which corresponds to $\sim 14 \%$. For channels on the same ASIC, the spread is $8 \%$, which is good enough for autotrigger operation with the same threshold for all channels.

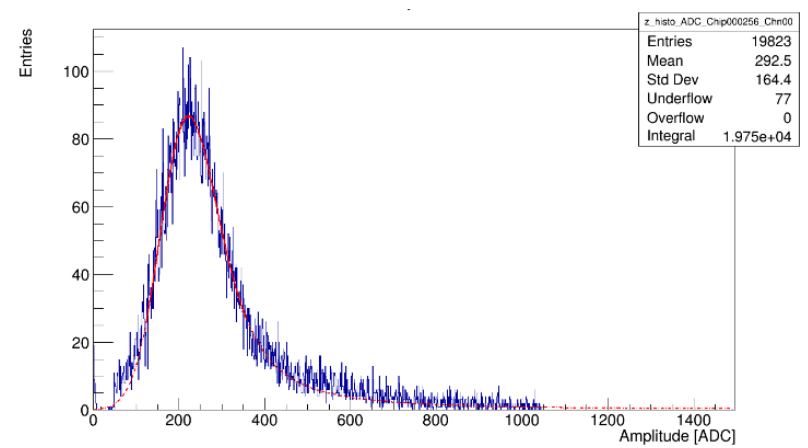

Fig. 5. Energy deposited by a muon in one AHCAL cell. The red solid line is obtained by fitting a Landau convoluted to a Gaussian function to the data. The dotted red line is the function extrapolated outside the fitting range.

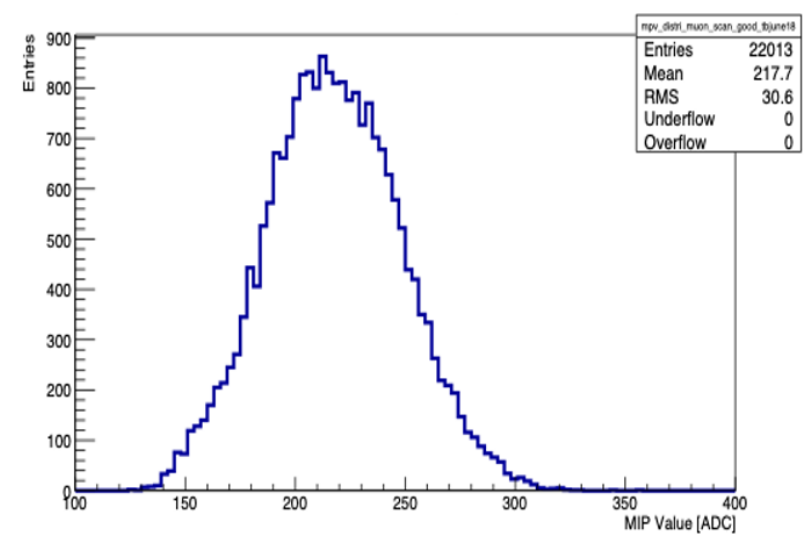

Fig. 6. MIP constant extracted for all AHCAL channels.

\section{Inter-Gain Calibration}

The SPIROC2E chip can store the ADC either in low gain (LG) or in high gain (HG) mode. The inter-gain calibration factor of the channel is the ratio of $\mathrm{HG}$ to $\mathrm{LG}$ and is required for smooth transition between HG and LG hit energies. This constant depends on ratio of capacitors because of the circuit design. The extraction of the inter-gain calibration coefficients from LED runs is possible because the range of linear response of the chip in both modes overlaps. The amplitude of the signal is varied within the linear range of $\mathrm{HG}$ and LG by varying the LED light intensity. The inter-calibration coefficient for each channel is taken as the slope of a linear fit as shown in Fig 7 . The inter-calibration factor ranges between 17 and 21 with a mean of 19.4 and a RMS spread of 0.66 which is $\sim 3 \%$. For the remaining channels the inter calibration values were taken as the average of the channels of the corresponding chip.

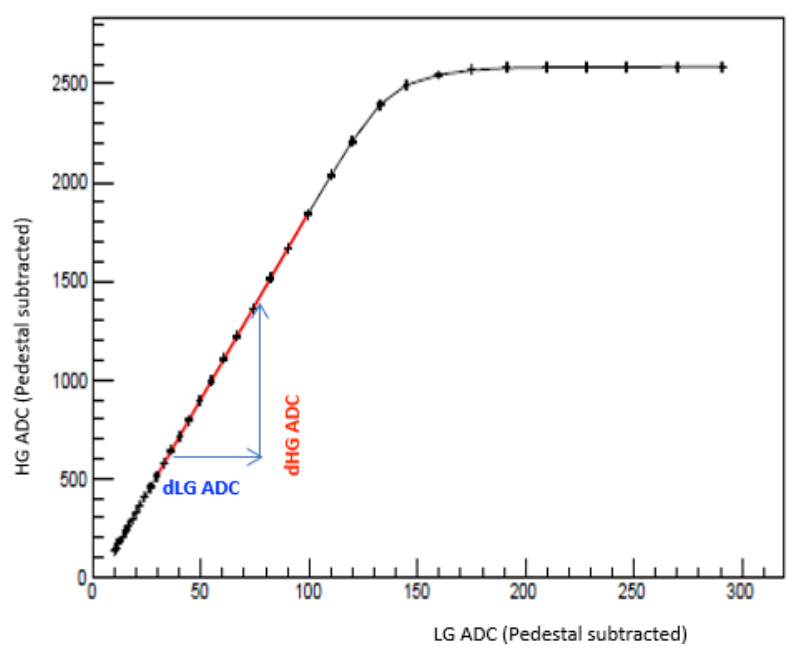

Fig. 7. Response of one AHCAL channel in the two amplifier gain modes (Low Gain and High Gain) for increasing LED light intensity.

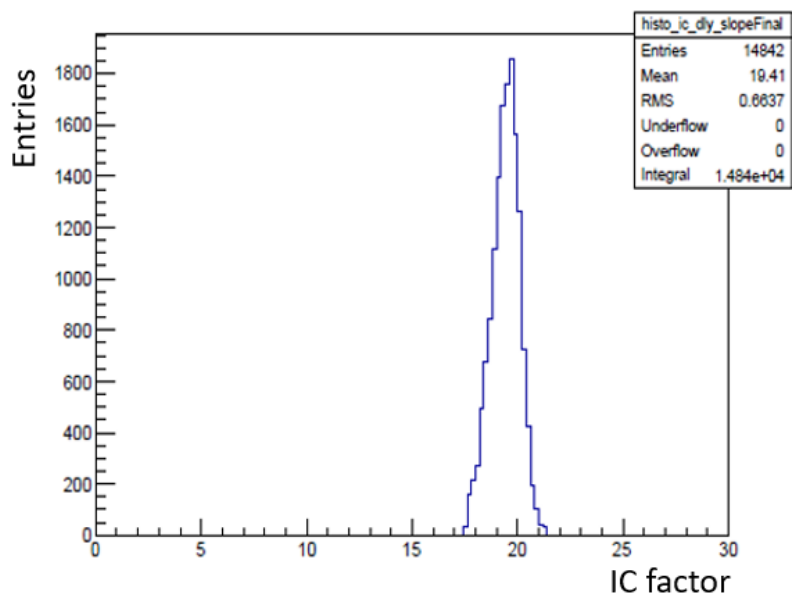

Fig. 8. Inter-gain calibration values extracted for all calibrated channels.

\section{SiPM Saturation}

Due to the limited number of pixels and the finite pixel recovery time, the SiPM is an intrinsically a non-linear device and limited by response saturation at high amplitudes. The response function of a SiPM describes the number of fired pixels as a function of the number of incoming photons (see figure 9p. It can be approximated by

$$
N_{\text {fired }}^{\text {sat }}=N_{\text {eff }} \quad\left(1-e^{N_{\text {fired }}^{\text {unsat }} / N_{\text {eff }}}\right),
$$

where $N_{\text {fired }}^{s a t}$ is the number of fired pixels and $N^{e f f}$ is the effective number of pixel in the SiPM. For studies of saturation behaviour bare SiPMs were used. A dedicated setup was used to ensure that all the pixels were illuminated in a homogeneous way. The $N_{\text {eff }}$ for MPPC S13360-1325PE is found to be consistent with the real number of pixels of 2668[7]. Alternatively, $N_{\text {eff }}$ has been extracted using the 
AHCAL measurements with the SiPM mounted on a tile, showing consistent results. In this case, also saturation effects in the ASIC can play a role. For the reconstruction of hit energies from the SiPM signals the inverse of the saturation function is used.

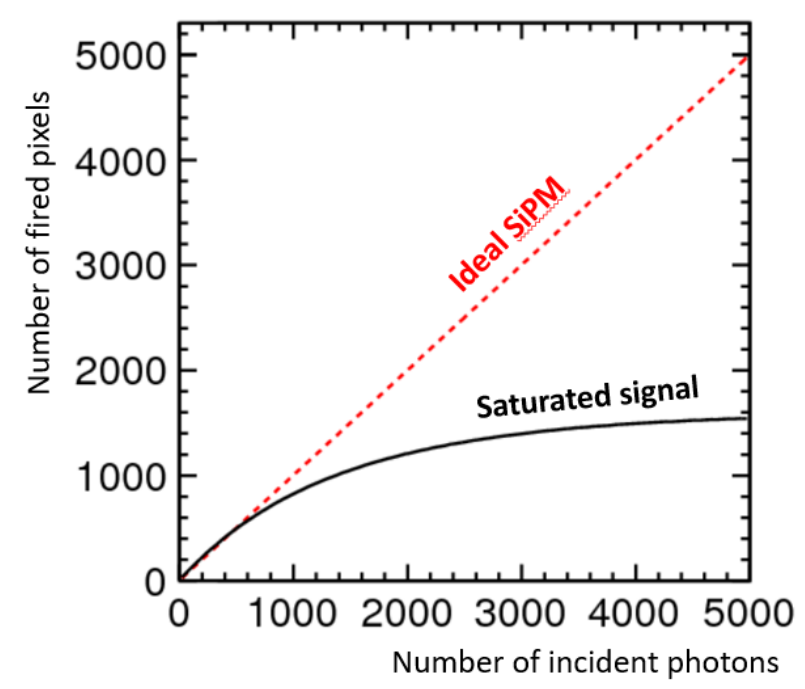

Fig. 9. Describing the response of SiPM.

\section{E. Time Calibration}

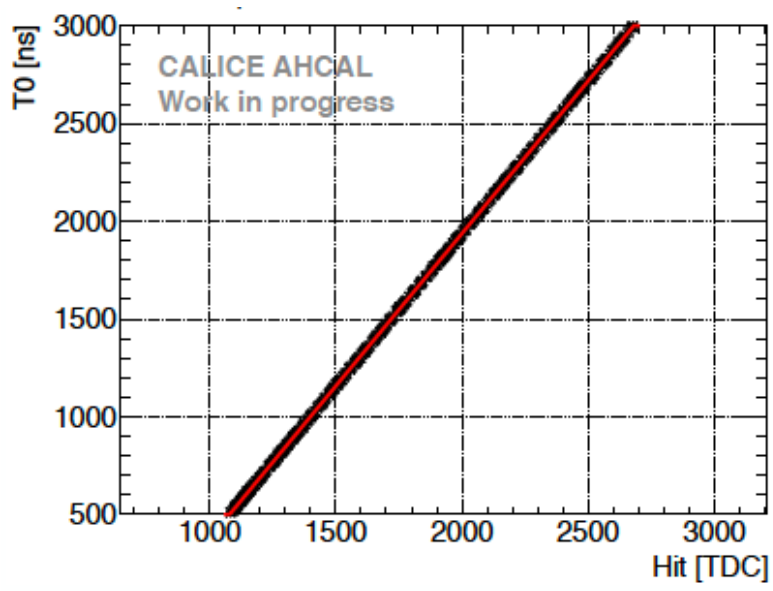

Fig. 10. TDC vs. time reference plot.

The time calibration is performed using muon runs. The time information is stored in TDC values and needs to be calibrated to nanoseconds to have a common time reference in each event. To record time, TDC voltage is ramped up and down in intervals of $4000 \mathrm{~ns}$. For this purpose the SPIROC2E chip has two TDC ramps for even and odd bunch crossings (bx) that form together a combined up and down ramping. Once a hit occurs the bxID as well as the current TDC value is recorded. From the slope of the TDC ramp and its pedestal the time in nanoseconds is calculated for both channel wise and memory cell-wise as shown in Fig 10. A detailed description on how the time calibration is performed can be found in [8].
A 1 ns time resolution of the AHCAL is one of the design goal for testbeam mode. In practice the time resolution is limited by the front-end electronics.

\section{CONCLUSION}

The CALICE AHCAL is a SiPM-on-tile steel sampling calorimeter prototype which handles large number of SiPMs ( $\sim 22000$ channels in total). It has been calibrated with an integrated LED system for SiPM gain, using minimum ionising particles (muons) for energy scale and corrected for the nonlinearity of the SiPM response curves. The detector has been successfully operated in test beams from May to October 2018 at the CERN SPS and was stable with more than $99.9 \%$ channels working. With the calibrated prototype, shapes and structures of hadronic showers as well as the separation of two close-by showers can be studied in great detail, thus testing directly an important ingredient to the performance of particle flow algorithms.

\section{ACKNOWLEDGMENT}

I would like to thank my colleagues from AHCAL group for allowing me to present material and results from their research and colleagues from the CALICE collaboration for their help.

\section{REFERENCES}

[1] M. Thomson, Particle Flow Calorimetry and the PandoraPFA Algorithm, Nucl. Instrum. Meth., vol. A611, pp. 2540, 2009.

[2] C. Adloff et al., Construction and Commissioning of the CALICE Analog Hadron Calorimeter Prototype, JINST 5 (2010) P05004, arXiv:1003.2662

[3] M. Reinecke Performance of the Large Scale Prototypes of the CALICE Tile Hadron Calorimeter, 2013 IEEE Nuclear Science Symposium and Medical Imaging Conference (2013 NSS/MIC), pp 15 ISSN 1082-365

[4] M. Bouchel, S. Callier, F. Dulucq, J. Fleury, J J. Jaeger, C. de La Taille, G. Martin-Chassard and L. Raux, SPIROC (SiPM Integrated Read-Out Chip):dedicated very front-end electronics for an ILCprototype hadronic calorimeter with SiPM read-out, 2011 JINST 6 C01098

[5] Y. Liu et al., Design of scintillator tiles read out by surface-mounted SiPMs for a future hadron calorimeter, IEEE Nucl. Sci. Symp. Med. Imag. Conf. Rec. (2014) [arXiv:1512.05900]

[6] Y. Liu al., 2014 IEEE Nuclear Science Symposium and Medical Imaging Conference and 21st Symposium on Room-Temperature Semiconductor Xray and Gamma-ray Detectors (NSS/MIC 2014 /RTSD 2014), Seattle, WA, USA, November 8-15, 2014 (Preprint arXiv:1512.05900)

[7] Q. Weitzel et al., "Measurement of the response of Silicon Photomultipliers from single photon detection to saturation", Nucl. Instrum. Meth., A, vol. 936, 21 August 2019, pages 558-560

[8] E. Brianne, Time Development of Hadronic Showers in a Highly Granular Analog Hadron Calorimeter. PhD thesis, University of Hamburg, 2018. 